\title{
Vibrational density of states of selenium through the glass transition
}

\author{
D. Caprion and H. R. Schober ${ }^{\text {a) }}$ \\ Institut für Festkörperforschung, Forschungszentrum Jülich, D-52425 Jülich, Germany
}

(Received 25 September 2000; accepted 1 December 2000)

\begin{abstract}
We present an investigation of the vibrational density of states (VDOS) of selenium calculated using two different methods. First we compute the VDOS from the velocity autocorrelation and study its evolution with the temperature. Secondly we compute it from the dynamical matrix. This latter method leads to imaginary frequencies for finite temperatures. In the literature these frequencies are called unstable and are related to the diffusion and/or the anharmonicity. We investigate the character of these frequencies, asking whether they are localized or extended, more acoustic or optic. We show that, below a temperature-dependent threshold frequency, they are similar to their real low-frequency counterparts. The temperature evolution of the number of these modes changes at the glass transition. (C) 2001 American Institute of Physics. [DOI: 10.1063/1.1343074]
\end{abstract}

\section{INTRODUCTION}

Amorphous selenium is one of the few elementary glasses. Its glass transition temperature, $T_{g} \approx 305 \mathrm{~K}$, is near room temperature which facilitates experiments. It has been studied extensively in experiments on both structural ${ }^{1-3}$ and dynamical properties. ${ }^{4-7}$ There are only few simulations ${ }^{8}$ on the dynamics of Se when it is quenched through the glass transition.

On the other hand, simulations on model systems have been done for some years where the vibrational density of states (VDOS) is computed in the hot and the supercooled liquid. $^{9-12}$ These simulations were aimed to link the vibrational and diffusional properties. ${ }^{13-15}$ Particular attention was given to the behavior of the instantaneous normal modes (INM). These INMs are gained by a generalization of the normal mode analysis by means of the dynamical matrix, as done routinely for crystals. The INM analysis in the liquid, as well as in the glass for $T>0 \mathrm{~K}$, leads to modes with negative eigenvalues, $(2 \pi \nu)^{2}$. The frequency, $\nu$, is imaginary. These modes are, therefore, sometimes referred to as imaginary modes, also called unstable modes, and are related either to the diffusion and/or to anharmonicity. Based on the soft potential model, ${ }^{16}$ the INMs were analyzed to find the threshold between the diffusive and the anharmonic domains of these unstable modes. ${ }^{17}$

The aim of this work is to investigate, for a realistic model of a glass, the evolution of the INMs upon quenching through the glass transition. Initially we want to see whether different domains exist in the INM-spectrum of Se and secondly, if so, whether it is possible to discriminate between diffusive and anharmonic domains of the INMs modes.

This paper is set out as follows. First we describe the method used to obtain the different configurations in the liquid, the super-cooled liquid and the glass. We then briefly recall different methods to obtain the VDOS. In Sec. III the VDOS of selenium at different temperatures are presented. In the next section the spectra are analyzed in terms of

${ }^{a)}$ Electronic mail: h.schober@fz-juelich.de acoustic- and opticlike modes, and of extended and localized modes. We correlate the change of the INMs with the glass transition and compare it with the temperature dependence of the diffusion constant. Finally we conclude and summarize this work.

\section{SIMULATIONS}

We did classical molecular dynamics simulations on a system of $N=2000$ atoms interacting via a three-body potential. This potential was constructed to reproduce the properties both of small clusters of Se and also of the trigonal crystalline phase. More details on the potential are given in Ref. 18. The potential has previously been used to study vibrations ${ }^{19}$ and local relaxations ${ }^{20,21}$ in amorphous Se. It has also been used to study the microscopic and electronic structure of $\mathrm{Se}^{22}$

In our simulations we used a Parrinello-Rahman algorithm ${ }^{23,24}$ to keep the pressure constant, equal to zero. The temperature was kept constant by rescaling the velocities at each integration step. The simulations were done in a cubic box with periodic boundary conditions.

The equations of motion were integrated using the velocity Verlet algorithm. ${ }^{25}$ Taking care of the stability of the algorithm, we used time steps of $1 \mathrm{ps}$ in the liquid, $2 \mathrm{ps}$ in the glass phase, and $4 \mathrm{ps}$ for the lowest temperature $(6 \mathrm{~K})$.

The samples in the liquid and super-cooled liquid phases were obtained by cooling well equilibrated liquids to the measurement temperatures. Subsequently the samples were aged during 800000 time steps (i.e., 800 ps). The samples in the glassy phase (i.e., $T \leqslant 290 \mathrm{~K}$ ) were derived by cooling the samples aged at $T=290 \mathrm{~K}$ to the desired measurement temperatures. The quench rates for theses two steps were equal, $Q_{r}=10^{13} \mathrm{~K} / \mathrm{s}$. The samples in the glassy phase were also aged for 800000 time steps $(1.6 \mathrm{~ns}$ for $T>6 \mathrm{~K}$ and $3.2 \mathrm{~ns}$ at $T=6 \mathrm{~K})$ before the measurements were taken.

To improve the statistics, we used four independent starting configurations in the liquid. After aging these different samples, we used in the measurements three different configurations of each of the independent samples. These 
configurations were picked during the simulation at constant temperature every 100000 time steps. We, therefore, had 12 different configurations for each temperature, assuming that 100000 time steps are enough to cancel correlations between configurations extracted from the molecular dynamics simulations.

In the previous simulations ${ }^{21}$ we estimated the glass transition temperature as $T_{g} \approx 300 \mathrm{~K}$ which is in good agreement with the experimental value $T_{g}=305 \mathrm{~K}$. One should notice that the effective quench rate for the samples is less than $10^{10} \mathrm{~K} / \mathrm{s}$ near $T_{g}$.

\section{METHODS}

Several methods exist to compute the vibrational density of states (VDOS) from molecular-dynamics simulations. One can use the method proposed by Beeman and Alben, ${ }^{26}$ or slightly modified by Oligschleger and Schön. ${ }^{19}$ This method is based on the solution of the equation of motions with the forces given in harmonic approximation.

Another method is to compute the velocityautocorrelation function $(\mathrm{VAF})^{27}$

$$
C_{v v}(t)=\left\langle\mathbf{v}\left(t+t_{0}\right) \cdot \mathbf{v}\left(t_{0}\right)\right\rangle_{t_{0}},
$$

and then to obtain the VDOS by a Fourier transform of the VAF

$$
Z(\omega)=\frac{1}{2 \pi} \int_{0}^{\infty} C_{v v}(t) \cos (\omega t) .
$$

This method also can be used in the liquid phase where the VAF additionally gives information about the diffusion. It should be noted that this method goes beyond the harmonic approximation and thus gives an insight into the anharmonicity.

A third method is to compute the $3 N \times 3 N$ dynamical matrix $\mathbf{D}$ (with $N$ the number of particles in the simulation)

$$
D_{i j}^{\alpha \beta}=\frac{1}{\sqrt{M_{i} M_{j}}}\left(\frac{\partial^{2} V}{\partial r_{i}^{\alpha} r_{j}^{\beta}}\right)_{\left\{\mathbf{r}_{i}^{0}\right\}},
$$

where $V$ is the interatomic potential, $M_{i}$ the mass of particle $i$ and $\mathbf{r}_{i}^{0}$ denotes the current configuration. The roman indices run over particles and the greek ones over the spatial directions. Diagonalizing the dynamical matrix one obtains the wanted information on the dynamics. For each mode of vibration $s$ the eigenvalue, $\lambda_{s}$, is related to the frequency of the vibration by $\lambda_{s}=\left(2 \pi \nu_{s}\right)^{2}$. The eigenvector coordinates $\mathbf{e}_{s}^{i}$ represent the vectorial amplitude of atom $i$ in the vibrational eigenmode.

Originally developed for the solid phase, this method has been transferred to the liquid and super-liquid phases in recent years. In an ordered solid far from a phase transition the energy landscape can be approximated by a quadratic function and, therefore, all eigenvectors, $\lambda_{s}$, are positive or zero. The three zero eigenvalues correspond to the three degrees of translation. The corresponding rotations are usually eliminated by the periodic boundary conditions. In amorphous or liquid samples the system is not necessarily in a quadratic minimum. For instance, if one atom at a given instance

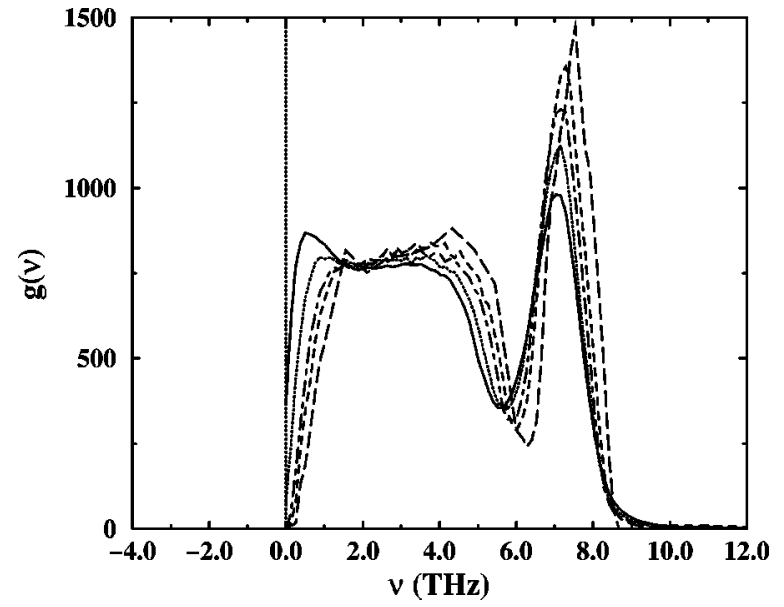

FIG. 1. Vibrational densities of states of liquid and amorphous Se obtained by Fourier transform of the velocity autocorrelation function at different temperatures: 6 K (long dashed), $200 \mathrm{~K}$ (short dashed), $290 \mathrm{~K}$ (dasheddotted), $445 \mathrm{~K}$ (dotted), and $495 \mathrm{~K}$ (solid line).

jumps over a barrier then the curvature of the energy landscape at this instantaneous configuration is not positive in all directions. Therefore, at least one eigenvalue of the dynamical matrix is negative, and the corresponding frequency, $\nu_{s}$, is imaginary. But this is not the only case where one obtains an imaginary frequency. Also a simple anharmonic well in the landscape energy can also lead to an imaginary frequency (see for instance Ref. 9). It is sufficient that the instantaneous configuration is beyond a turning point of the multidimensional energy landscape.

In this work we will focus on this dynamic matrix method. Thanks to the knowledge of the eigenvectors, this method can provide detailed information on the vibrations, especially on the nature of the modes. It can help to answer questions, such as whether the unstable modes are acousticlike or opticlike, extended or localized. For comparison we also calculate the VDOS from the velocity autocorrelation function, Eq. (2).

\section{VIBRATIONAL DENSITY OF STATES}

To study the change of the VDOS with temperature we show the densities of states obtained by the two methods for different temperatures, above and below the glass transition temperature.

Figure 1 presents the VDOS obtained from the Fourier transform of the VAF, Eq. (2). Two bands are clearly distinguished. The peak at high frequencies, above $6 \mathrm{THz}$, corresponds to the intramolecular bond stretching vibrations. The broad band below $6 \mathrm{THz}$ is due, both to bond bending modes and to the intermolecular modes, at the lower frequencies. The shapes and the values of these two bands are similar to the ones measured in experiment. ${ }^{28}$ There is a marked improvement compared to the results of the earlier simulation using a much simpler model. ${ }^{8}$

With increasing temperature the spectrum as a whole shifts to lower frequencies. Additionally, above $T=T_{g}$ a peak appears at the lowest frequencies. This peak is the signature of diffusion which causes a quasi elastic peak over- 


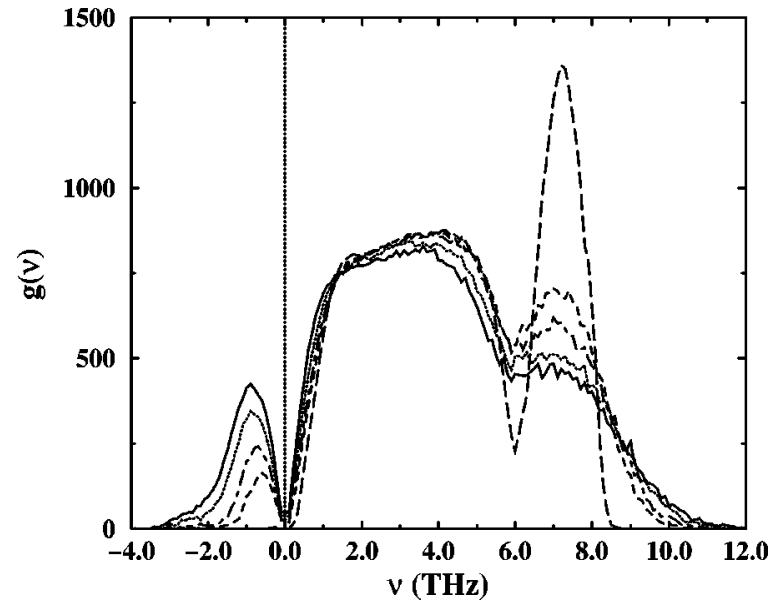

FIG. 2. Vibrational density of states computed from the dynamical matrix at different temperatures: $6 \mathrm{~K}$ (long dashed), $200 \mathrm{~K}$ (short dashed), $290 \mathrm{~K}$ (dashed-dotted), $445 \mathrm{~K}$ (dotted), and $495 \mathrm{~K}$ (solid line).

laying the VDOS. This effect is also observed in experiment. The rest of the lower-frequency band does not change significantly when the temperature is increased. The highfrequency band broadens with temperature and consequently its peak height decreases.

Figure 2 shows for the same set of temperatures the VDOS obtained from the diagonalization of the dynamical matrix, i.e., the spectrum of the instantaneous modes. As usual we multiply the imaginary frequencies by $i$ and show them as negative frequencies on the same axis as the real ones. We now have three bands instead of the two in Fig. 1. The extra band corresponds to the imaginary frequencies and is, therefore, not present with the VAF method. Secondly, contrary to Fig. 1, the low-frequency band $(0-6 \mathrm{THz})$ does not shift with increasing temperature whereas the highfrequency band changes strongly. While the position of its maximum shifts only slightly to lower values, its maximum value decreases dramatically with increasing temperature. Simultaneously its width increases. Clearly the imaginary band grows rapidly upon heating the system. This behavior had been reported before from several numerical simulations of other materials. It is related to anharmonicity and diffusion, both of which gain in importance with increasing temperature. This effect would not be present in a quasi harmonic description where one takes a harmonic expansion from a temperature-dependent equilibrium configuration and all frequencies are real. Even if we neglect mixing of the quasiharmonic eigenmodes for the moment, the potential-energy curve as function of elongation in the eigenmode will be anharmonic and in some cases have a low-lying maximum. For some critical elongation the curvature goes through zero (turning point) and the eigenvalue of the mode becomes negative. The VDOS of the INM corresponds to a time average of the potential energy curve. With increasing temperature the probability to find elongations larger than the critical one rises and, therefore, the imaginary band of the VDOS grows. Anharmonic mixing between the modes will further increase this effect

The difference between Figs. 1 and 2 poses the question of whether the instantaneous mode spectrum shifts also to

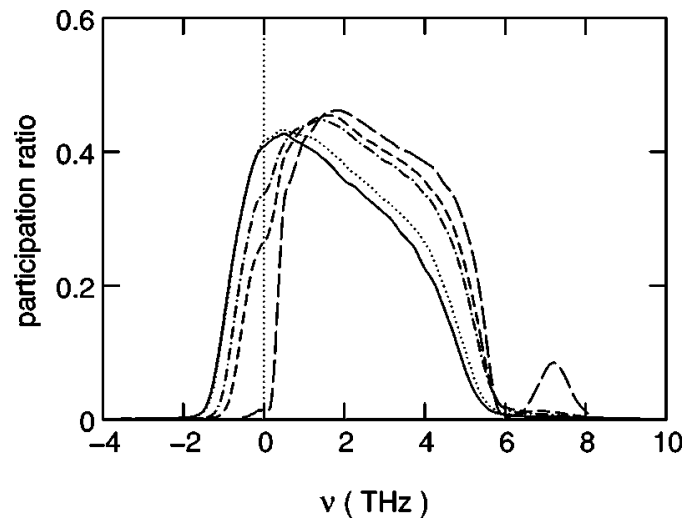

FIG. 3. Participation ratio of the eigenmodes of the instantaneous dynamic matrix at different temperatures: $6 \mathrm{~K}$ (long dashed), $200 \mathrm{~K}$ (short dashed), $290 \mathrm{~K}$ (dashed-dotted), $445 \mathrm{~K}$ (dotted), and $495 \mathrm{~K}$ (solid line).

lower frequencies with increasing temperature, as has been observed by the VAF-method in Fig. 1. In that case the nature of the instantaneous imaginary frequency modes should be similar to the one of the modes of the real low-frequency band. At the same time one might also expect that some of theses imaginary (unstable) modes are due to diffusion. With increasing temperature, the number of atoms jumping barriers will increase and this will be reflected in an increase of the number of imaginary modes. Therefore, two effects of temperature should occur simultaneously, a shift of real lowfrequency modes (due to the softening of the system) and an increase of diffusive imaginary modes. To distinguish between diffusive or anharmonic modes we studied their nature, using simple tools.

\section{NATURE OF THE MODES}

First we check whether the modes are localized or extended. A usual measure of localization is the participation ratio

$$
p(\nu)=\left\{N \sum_{i}\left(\mathbf{e}_{i}(\nu) \cdot \mathbf{e}_{i}(\nu)\right)^{2}\right\}^{-1} .
$$

For extended modes the participation ratio is of order unity. For localized modes it should scale inversely with the system size. This scaling behavior is, however, only observed at the band edges, for low-spectral densities. Two effects contribute to an increase of participation ratio even for modes which can, in some sense, be considered as localized. First, modes of similar frequency will interact if they are sufficiently close. With increasing system size this will give, instead of $p(\nu) \sim 1 / N$, an asymptotic behavior $p(\nu) \sim c(\nu)$ where $c(\nu)$ is the concentration of localized modes at frequency $\nu$. Second, for low frequencies, the interaction of low-frequency quasi-localized modes and sound waves becomes important. This is well known from studies of resonant vibrations in defect crystals.

Let us discuss the low temperature behavior of the participation ratio, long-dashed line in Fig. 3. The above discussed effects are reflected in the steep increase at low frequencies of $p(\nu)$. For our system size, the transverse soundwave with the largest wave length, compatible with 
periodicity, has a frequency of $\nu \approx 0.4 \mathrm{THz}$. Therefore, the participation ratios shown in Fig. 3 correspond to an infinite matrix for $\nu>0.4 \mathrm{THz}$, whereas for lower frequencies there will be size effects. The bulk of the modes in the lowerfrequency band is extended with $p(\nu) \approx 0.4$, slowly falling with increasing $\nu$. Truly localized modes are observed in the gap between the two frequency bands and at the highest frequencies. This is again similar to the observations in defect crystals. The low values of $p(\nu)$ for the higher-frequency band is a typical effect of amorphicity. It reflects the breakup of the infinite chains of the crystal into shorter chains and rings in the amorphous structure. ${ }^{21}$

With increasing temperature the modes of the highfrequency band localize even more, $p(\nu) \rightarrow 1 / N$. The chain structure weakens and can no longer support extended bond stretching vibrations. Below $6 \mathrm{THz}$ the $p(\nu)$ curve shifts with increasing temperature to the left (into the imaginary frequency part). The drop at the higher frequencies becomes more pronounced. We will see from the following that this can be attributed to an increased mixing between the two frequency bands.

From a study of a soft sphere model glass it has been suggested that the increase of $p(\nu)$ at imaginary frequencies with temperature can be used as an indicator of the glass transition. ${ }^{9}$ The glass transition is related to the temperature where truly unstable modes become extended. Without an extensive investigation it is not possible to distinguish those modes (indicating a motion over a barrier) from strongly anharmonic vibrations. But one hopes to find a limiting imaginary frequency below which modes are mostly unstable. Due to the inherent chain structure, the extended modes have much lower participation ratios in Se, $p(\nu)$ $\approx 0.4$, than in the closed packed simple soft sphere glass where the corresponding values are $p(\nu) \approx 0.55$. As a consequence one cannot distinguish extended and weakly localized modes unambiguously using only $p(\nu)$ as criterion. Keeping this caveat in mind, we find that the glass transition occurs when the average $p(\nu \approx 0)$ increases to 0.35 . Single modes with $p>0.35$ are observed around $\nu=-0.3 \pm 0.1$ Thz.

As we have seen, the participation ratio is not sufficient to classify the modes. Therefore, we test the modes for additional properties. As a first step we check whether the modes are of a more acoustic or optic type. For this, we introduce a phase parameter $\phi$ defined as ${ }^{29-33}$

$$
\phi(\nu)=\frac{\sum_{\langle i, j\rangle} \mathbf{e}_{i}(\nu) \cdot \mathbf{e}_{j}(\nu)}{\sum_{\langle i, j\rangle}\left|\mathbf{e}_{i}(\nu) \cdot \mathbf{e}_{j}(\nu)\right|},
$$

where $\langle i, j\rangle$ indicates nearest neighbors. This definition ensures that for more acoustic vibrations the phase parameter $\phi(\nu) \approx 1$ (the vibrations of neighboring atoms are in phase), while it is $\phi(\nu) \approx-1$ for an optic (anti-phase) vibration.

In Fig. 4, we show the phase parameter for three different temperatures above, near and below the glass transition temperature. To clarify the physics the curves have been smoothed. The values of $\phi(\nu)$ scatter strongly at the edges of the spectra, especially at very high frequencies around 8 Thz, indicating that the parameter loses its meaning for these vibrations. We have seen that the modes are strongly local-

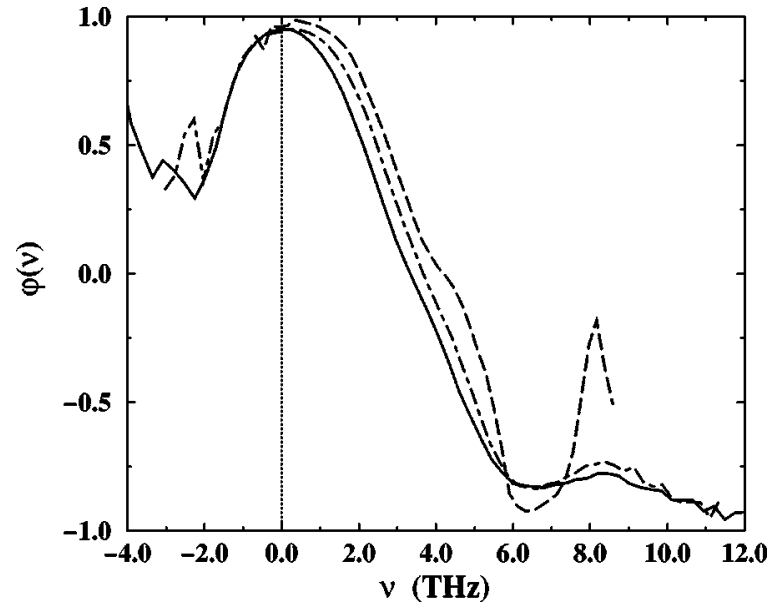

FIG. 4. Phase parameter $\phi(\nu)$ at different temperatures: $T=6 \mathrm{~K}$ (dashed line), $T=290 \mathrm{~K}$ (dotted-dashed line), and $T=495 \mathrm{~K}$ (solid line).

ized in this frequency range and, therefore, the following analysis which is based on a plane-wave behavior of the modes, is no longer applicable. The same holds, to a lesser extent, for the edge of the spectrum at imaginary frequencies, i.e., around $-4 i$ Thz.

We first look at the low temperature values $(T=6$ $\mathrm{K}$-dashed line in Fig. 4). As to be expected, the lowfrequency modes are predominantly acoustic. With increasing frequency, $\phi$ decreases to nearly -1 at the frequency of the gap in the spectrum, i.e., around $6 \mathrm{THz}$. In the high frequency band, the phase parameter increases again to reach a value of zero before it drops again. This last decrease is probably an artifact of the smoothing procedure. As said above, the values for the single modes scatter strongly. The same increase of the phase parameter in the high-frequency band is observed in the VDOS of a crystalline configuration of trigonal Se. This structure consists of parallel helical chains with periodicity three. For a $q=0$ longitudinal mode along a chain one gets $\phi \approx-0.2$. At this temperature, there are only few imaginary modes and these seem to have the same characteristics as the ones with very low real frequencies, i.e., they are acousticlike.

With increasing temperature, the evolution of $\phi$ shows some interesting features. First, when the system is heated there is a general shift of the $\phi(\nu)$ curve to lower frequencies. This is expected from the shift of the whole spectrum with increasing temperature seen in Fig. 2. Second, $\phi$ in the high-frequency band is more or less equal for both higher temperatures. Third, in the imaginary frequency range the phase parameter starts from $\approx 1$ at $0 \mathrm{THz}$ and decreases before it starts to scatter strongly. This behavior is seen below and above $T_{g}$. We can, therefore, conclude that the imaginary modes, in the range of small imaginary values near 0 Thz, are acousticlike while it is more difficult to characterize their nature in the range of high imaginary values.

Analogously to the phase parameter, we define a bond stretch parameter $S(\nu)^{32}$

$$
S(\nu)=\frac{\sum_{\langle i, j\rangle}\left|\left(\mathbf{e}_{j}(\nu)-\mathbf{e}_{i}(\nu)\right) \cdot \hat{r}_{i j}\right|}{\sum_{\langle i, j\rangle}\left|\left(\mathbf{e}_{j}(\nu)-\mathbf{e}_{i}(\nu)\right)\right|},
$$




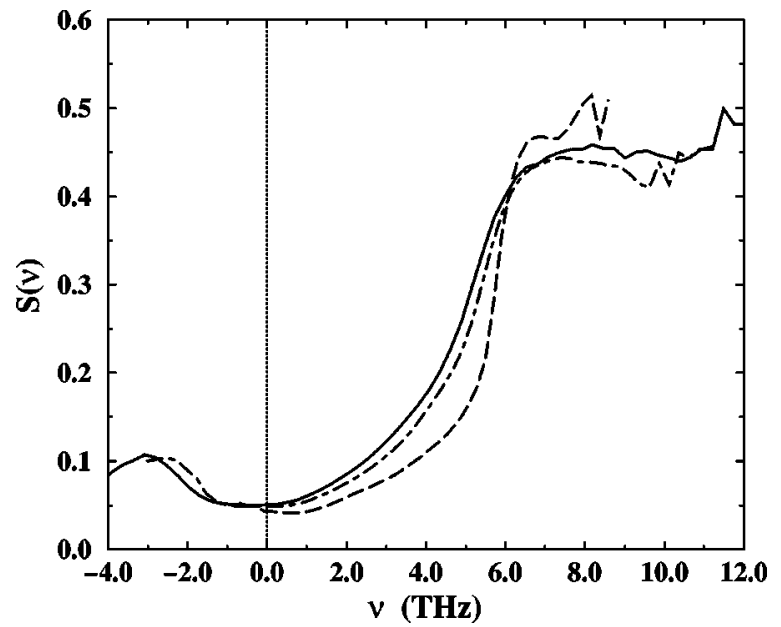

FIG. 5. Stretching parameter $S(\nu)$ at different temperatures:6 K (dashed line), $290 \mathrm{~K}$ (dashed-dotted line), and $495 \mathrm{~K}$ (solid line).

where $\hat{r}_{i j}$ is a unit vector in the direction of the bond from atom $i$ to atom $j$. This bond stretching parameter is so defined, that it is equal to one for a mode that only involves stretching of the bonds. It is equal to zero when all motion is orthogonal to the bonds.

Figure 5 reports the bond stretching parameter for the same three temperatures as used in Fig. 4 for the phase parameters. The curves are again smoothed to avoid spurious effects at the edges. Taking first the low-temperature curve (6 K, dashed line) one sees that, as expected, the lowfrequency band is mainly due to modes that do not strongly involve bond stretching. Contrarily the high-frequency band consists of bond stretching modes. This is in full agreement with experiments. ${ }^{34}$

With increasing temperature, we observe the same trends as above, e.g., a general shift of the curves to smaller frequencies. Moving to imaginary frequencies the stretching parameter changes continuously and differs only slightly from the values at low real frequencies. At the largest imaginary frequencies, $\nu<-3 i \mathrm{THz}, S(\nu)$ again scatters, but not as strongly as the phase parameter, Fig. 4.

From the above we can so far conclude that, as to be expected, the lower band of the instantaneous mode spectrum shifts to lower frequencies when the system is heated to the liquid phase. Furthermore the modes with imaginary frequencies have the same character as the ones with low real frequencies. Therefore, we can now answer the first of our posed questions. The modes with imaginary frequencies, at least the ones with not too large absolute frequencies, originate from the lowest frequency part of the real spectrum. For the largest imaginary frequencies their character is, however, no longer as well defined.

The above analysis was based on concepts strictly valid for wavelike modes only. It is, therefore, understandable that it is not really applicable to the modes with the largest imaginary frequencies, since these modes are no longer extended.

In order to clarify this last point, we introduce a third tool, namely the correlation length or gyration radius. We define it by ${ }^{32,35}$

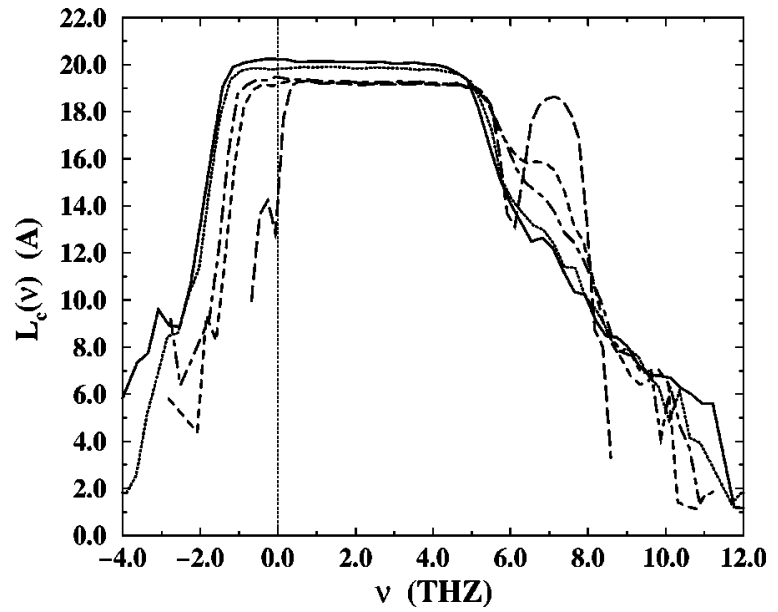

FIG. 6. Correlation length $L_{c}(\nu)$ at different temperatures: $6 \mathrm{~K}$ (long dashed), $200 \mathrm{~K}$ (short dashed), $290 \mathrm{~K}$ (dashed-dotted), $445 \mathrm{~K}$ (dotted), and $495 \mathrm{~K}$ (solid line).

$$
L_{c}^{2}(\nu)=\sum_{i}\left|\mathbf{r}_{i}-\mathbf{r}_{g}(\nu)\right|^{2}\left|\mathbf{e}_{i}(\nu)\right|^{2},
$$

where $\mathbf{r}_{g}(\nu)$ is the center-of-mass of the mode, defined by

$$
\mathbf{r}_{g}(\nu)=\sum_{i} \mathbf{r}_{i}\left|\mathbf{e}_{i}(\nu)\right|^{2}
$$

The gyration radius measures the spatial extent of a mode. If the mode is extended (plane wavelike) the correlation length $L_{c}$ tends to $L_{\text {box }} / 2$ where $L_{\text {box }}$ is the size of the simulation box; in the case of a localized mode this length goes to zero.

The definition of the gyration radius is not unique. In particular, one can change the weight in the definition of $L_{c}$ from $\left|\mathbf{e}_{i}(\nu)\right|^{2}$, corresponding to effective masses, to $\left|\mathbf{e}_{i}(\nu)\right|^{4}$, corresponding to participation ratios. ${ }^{35}$ In the latter case the absolute value of $L_{c}$ changes but the qualitative behavior remains the same.

In Fig. 6 we plot the average correlation length as function of frequency for the same temperatures as in Figs. 1 and 2 , both below and above $T_{g}$. To show the essential features more clearly, also these curves have been smoothed. Comparing with Fig. 3 where the participation ratios were shown one observes a similar structure which is now, however, much more pronounced.

As in the previous discussion of $\phi$ and $S$, we first focus on the lowest temperature, namely $6 \mathrm{~K}$. At this temperature the value of $L_{c}$ tends to a constant value of about $19 \AA$ in both the lower and upper band. The size of the simulation box at $6 \mathrm{~K}$ is $19.23 \AA$. Therefore, we deduce that both bands are formed by modes extended over the whole volume. It is also evident that $L_{c}$ drops at the upper and lower edges of the spectrum and at the gap, which indicates that both the modes at the lowest and highest frequencies and in the gap are localized. As regards the lowest frequencies we want to recall that localization is weak. In an infinite system these modes interact with the sound waves, forming resonant (quasilocalized) modes, see e.g., Ref. 35. Due to the finite size of the simulation volume long wavelength phonons are excluded and the resonant modes show up as localized. 


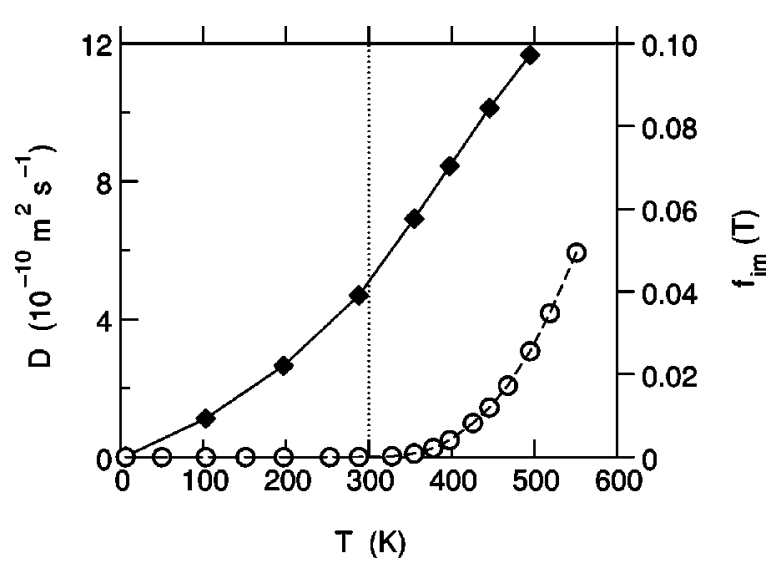

FIG. 7. Fraction of imaginary frequency modes $f_{\text {im }}$, (full diamonds) and diffusion constant (circles) as function of temperature. The lines are guides to the eye only. The glass transition temperature, $T_{g} \approx 300 \mathrm{~K}$, is indicated by the dotted line.

Several effects are seen when the temperature is increased. First, as already seen for $\phi$ and $S$, there is an overall shift in frequency. Second, the modes with lowest real frequencies are extended at all temperatures. The marginal increase of the $L_{c}$ value reflects only the volume expansion with temperature. In contrast, as already observed in the participation ratio, the high frequency band localizes more and more with increasing temperature. As discussed above, this band is formed by the intra-chain vibrations and with increasing temperature the chains get broken and the modes localize. Third, there is a strong shift in the imaginary frequency range. In contrast to Fig. 3, there is a well defined edge separating extended and localized modes. We observe that the modes with small imaginary frequencies (i.e., typically $\nu<1.0 i \mathrm{Thz}$ ) are extended modes. Thus, at all temperatures, continuing from zero frequency, the correlation length at first stays constant in a straight continuation from the real frequencies before dropping steeply. This drop gives a much clearer border between extended and localized behavior than the one defined by the participation ratio.

\section{GLASS TRANSITION AND DIFFUSION}

The temperature variation of the imaginary frequency modes is often related to the glass transition and the diffusion in the liquid. In Fig. 7 we plot the fraction of the modes with imaginary frequencies, $f_{\mathrm{im}}(T)$, as function of temperature. There is a clear change of temperature behavior at the glass transition temperature $T_{g}$. In the glassy state, $T<T_{g}$, $f_{\text {im }}(T)$ increases nonlinearly, whereas above $T_{g}$ it follows a law $f_{\text {im }}(T)=A+B^{*} T$. Seen the other way, this means that when the liquid configuration becomes partially arrested at the glass transition also unstable modes get frozen in. This reflects, e.g., the typical glassy effects such as local relaxations. The spectrum alone does not give information on the nature of these modes or whether they are homogeneously or heterogeneously distributed.

If one argues along the lines of Bembenek and Laird ${ }^{9}$ that the glass transition is reached when extended modes become unstable, then the correlation length of the modes, Fig. 6, fixes the threshold frequency separating unstable and anharmonic modes to $\nu_{\lim } \approx-0.5 i \mathrm{THz}$. However, at this frequency the average participation ratio, Fig. 3 , at $T=T_{g}$ is only $p(-0.5 i) \approx 0.29$ which would not allow one to identify these modes as extended. Taking Bembenek and Laird's conjecture and Figs. 3 and 4 we find that below the glass transition unstable modes are frozen in. These modes are local, have acoustic characteristics and do not involve bond stretching.

The fact that over barrier motion will lead to imaginary frequency modes suggests that their density is related to diffusion. Based on earlier ideas by Zwanzig ${ }^{36}$ it was suggested that the temperature dependence of the diffusion constant in the liquid should be dominated by the temperature dependence of $f_{\mathrm{im}} \cdot{ }^{37,38}$ The fact that imaginary frequency modes are also observed in crystals at elevated temperatures has lead to a challenge of this idea. ${ }^{15}$ Comparing the temperature variation of the diffusion constant and of $f_{\text {im }}$ in Fig. 7, we find that a proportionality is only given above $T \approx 400 \mathrm{~K}$, well above $T_{g}$. There is no proportionality between the two quantities in the supercooled liquid. This cannot be remedied either by using a cutoff frequency or by subtracting $f_{\mathrm{im}}\left(T_{g}\right)$. It is not clear how this breakdown of the proportionality in the supercooled region is related to the structure and which other factor entering into a calculation of the diffusion constant is strongly temperature dependent. Different from the closed packed structures studied before ${ }^{37} \mathrm{Se}$ forms, at not too high temperatures, rings and chains which partially break up with increasing temperatures. The chain structure might influence the temperature behavior of both, the diffusion constant and $f_{\text {im }}$. We see in neither of them an indication of a cross over from a chain to a single atom behavior. This does, however, not necessarily mean that there is no structural effect. Comparing our results with other work it should be kept in mind that we used constant temperature/constant pressure ensembles (NPT). The other authors have used constant volume (NVE) ensembles. Assuming that for these ensembles the proportionality would also be observed for Se, one would conclude that the volume dependence of the imaginary modes and of the diffusion constant differ strongly in supercooled liquids. This point should be investigated.

\section{CONCLUSION}

Using an effective three-body interatomic interaction, we computed the vibrational density of states of selenium as function of temperature, both in the liquid and amorphous states. We compared the results obtained from the velocity autocorrelation with the instantaneous mode spectrum. Both methods give similar results at low temperatures. When the system is heated, the whole spectra shift to lower frequencies. This reflects the over-all softening of the system with increasing temperature. In a second step we have investigated the structure of the modes obtained by diagonalizing the dynamical matrix, with special emphasis on the modes with imaginary frequencies. We have shown that the modes with small imaginary frequencies have the same nature as the real low-frequency modes, i.e., they are acousticlike and do not stretch the nearest-neighbor bonds. There is a transition from extended to localized modes which shifts with increas- 
ing temperature to larger imaginary frequencies. The temperature dependence of the fraction of modes which have imaginary frequency clearly changes at the glass transition. Following the idea that the glass transition occurs when extended modes become unstable we can identify a cutoff frequency separating purely anharmonic modes from unstable ones. The latter indicate motion from one configurational minimum to another. The character of the modes with large imaginary frequencies is more difficult to evaluate. They are usually identified as diffusive modes. Contrary to some theories, we think that the threshold between the diffusive and anharmonic (or extended) imaginary modes could depend on the temperature. This latter point should be investigated in the future in order to give an accurate estimation of this threshold, for instance by drawing the energy profile of these modes.

Contrary to other work, we do not find a proportionality between the fraction of imaginary frequency modes and the diffusion constant in the undercooled liquid regime. It remains to be seen whether this is typical for the type of material or is a result of the constant pressure ensemble chosen.

\section{ACKNOWLEDGMENTS}

We would like to thank C. Oligschleger for her help at the beginning of this work. One of us (D.C.) would like to thank the A. von Humboldt Foundation for its support.

${ }^{1}$ R. Kaplow, T. A. Rowe, and B. L. Averbach, Phys. Rev. 168, 168 (1968).

${ }^{2}$ F. Y. Hansen, T. S. Kundsen, and K. Carneiro, J. Chem. Phys. 62, 1556 (1975).

${ }^{3}$ W. D. Wei, B. W. Corb, and B. L. Averbach, J. Non-Cryst. Solids 53, 19 (1982); B. W. Corb, W. D. Wei, and B. L. Averbach, ibid. 53, 29 (1982).

${ }^{4}$ W. A. Philips, U. Buchenau, N. Nücker, A.-J. Dianoux, and W. Petry, Phys. Rev. Lett. 63, 2381 (1989).

${ }^{5}$ U. Buchenau and R. Zorn, Europhys. Lett. 18, 523 (1992).

${ }^{6}$ M. Foret, B. Hehlen, G. Taillades, E. Courtens, R. Vacher, H. Casalta, and B. Dorner, Phys. Rev. Lett. 81, 2100 (1998).

${ }^{7}$ F. J. Bermejo, M. García-Hernández, F. J. Mompeán, D. MacMorrow, and J. L. Martinez, Phys. Rev. B 51, 11932 (1995), and references therein.

${ }^{8}$ S. Balasubramanian, K. V. Damodaran, and K. J. Rao, Chem. Phys. 166, 131 (1992)

${ }^{9}$ S. D. Bembenek and B. B. Laird, Phys. Rev. Lett. 74, 936 (1995); J. Chem. Phys. 104, 5199 (1996).

${ }^{10}$ T.-M. Wu and S.-F. Tsay, J. Chem. Phys. 105, 9281 (1996); Phys. Rev. B 58, 27 (1998).
${ }^{11}$ M. Cho, G. R. Fleming, S. Saito, I. Ohmine, and R. M. Stratt, J. Phys. Chem. 100, 6672 (1994); E. F. David and R. M. Stratt, J. Chem. Phys. 109, 1375 (1998).

${ }^{12}$ R. L. Murry, J. T. Fourkas, W.-X. Li, and T. Keyes, J. Chem. Phys. 110, 10410 (1999); 110, 10423 (1999).

${ }^{13}$ G. Seeley and T. Keyes, J. Chem. Phys. 91, 5581 (1989); B. Mafan, T. Keyes, and G. Seeley, ibid. 92, 7565 (1990); 94, 6762 (1991); G. Seeley, T. Keyes, and B. Mafan, ibid. 95, 3847 (1991); T. Keyes, W.-X. Li, and U. Zürcher, ibid. 109, 4693 (1998).

${ }^{14}$ Yi Wan and R. M. Stratt, J. Chem. Phys. 100, 5123 (1994); M. Buchner, B. M. Ladanyi, and R. M. Stratt, ibid. 97, 8522 (1992); G. Goodyear and R. M. Stratt, ibid. 105, 10050 (1996); R. E. Larsen and R. M. Stratt, ibid. 110, 1036 (1999).

${ }^{15}$ R. Gezelter, E. Rabani, and B. J. Berne, J. Chem. Phys. 107, 4618 (1997).

${ }^{16}$ V. G. Karpov, M. I. Klinger, and F. N. Ignatiev, Sov. Phys. JETP 65, 1641 (1983); Yu. M. Galperin, V. L. Gurevich, and D. A. Parshin, Phys. Rev. B 32, 6873 (1985).

${ }^{17}$ U. Zürcher, T. Keyes, and B. B. Laird (unpublished).

${ }^{18}$ C. Oligschleger, R. O. Jones, S. M. Reimann, and H. R. Schober, Phys. Rev. B 53, 6165 (1996).

${ }^{19}$ C. Oligschleger and H. R. Schober, Physica A 201, 391 (1993); C. Oligschleger and J. C. Schön, J. Phys.: Condens. Matter 9, 1049 (1997).

${ }^{20}$ C. Oligschleger and H. R. Schober, Solid State Commun. 93, 1031 (1995); H. R. Schober, C. Gaukel, and C. Oligschleger, Prog. Theor. Phys. Suppl. 126, 67 (1997).

${ }^{21}$ D. Caprion and H. R. Schober, Phys. Rev. B 62, 3709 (2000).

${ }^{22}$ T. Koslowski, Z. Phys. Chem. (Munich) 210, 45 (1999).

${ }^{23}$ M. Parrinello and A. Rahman, Phys. Rev. Lett. 45, 1196 (1980)

${ }^{24}$ M. Parrinello and A. Rahman, J. Appl. Phys. 52, 7182 (1981).

${ }^{25}$ W. C. Swope, H. C. Andersen, P. Berens, and K. R. Wilson, J. Chem. Phys. 76, 637 (1982).

${ }^{26}$ D. Beeman and R. Alben, Adv. Phys. 26, 339 (1977).

${ }^{27}$ J. H. Dickey and A. Paskin, Phys. Rev. 128, 2589 (1969).

${ }^{28}$ F. Gompf, J. Phys. Chem. Solids 42, 539 (1981); L. M. Needham, M. Cutroni, A. J. Dianoux, and H. M. Rosenberg, J. Phys.: Condens. Matter 5, 637 (1993).

${ }^{29}$ R. J. Bell and P. Dean, Philos. Mag. 25, 1381 (1972).

${ }^{30}$ R. J. Bell and D. C. Hibbins-Butler, J. Phys. C 8, 787 (1975).

${ }^{31}$ W. Jin, P. Vashishta, R. K. Kalia, and J. P. Rino, Phys. Rev. B 48, 9359 (1993).

${ }^{32}$ M. Marinov and N. Zotov, Phys. Rev. B 55, 2938 (1997).

${ }^{33}$ S. N. Taraskin and S. R. Elliott, Phys. Rev. B 56, 8605 (1997).

${ }^{34}$ F. Gompf, J. Phys. Chem. Solids 42, 539 (1981); L. M. Needham, M. Cutroni, A. J. Dianoux, and H. M. Rosenberg, J. Phys.: Condens. Matter 5, 637 (1993).

${ }^{35}$ H. R. Schober and C. Oligschleger, Phys. Rev. B 53, 11469 (1996).

${ }^{36}$ R. Zwanzig, J. Chem. Phys. 79, 4507 (1983).

${ }^{37}$ T. Keyes, J. Phys. Chem. 101, 1921 (1997); T. Keyes, W.-X. Li, and U. Zurcher, J. Chem. Phys. 109, 4693 (1998).

${ }^{38}$ M. Ribeiro and P. A. Madden, J. Chem. Phys. 108, 3256 (1998). 